\title{
Empowerment-orientierte Kompetenzentwicklung auf der Grundlage von Mobile Assisted Seamless Learning
}

Sabine Seufert und Christoph Meier

\section{Zusammenfassung}

Mobile Learning wird in den letzten Jahren von Experten und Expertinnen immer wieder als Trend bezeichnet, die tatsächliche Nutzung und Verbreitung in der betrieblichen Bildung ist bislang jedoch eher ernüchternd. Neuere Ansätze im Bildungsmanagement sehen die Potenziale von Mobile Learning nicht nur in zeit- und ortsunabhängigem (Mikro-) Lernen, in der Verbindung von informellem und formellem Lernen, sondern auch bei der Unterstützung von Lernen entlang einer Bildungsbiographie über verschiedene Bildungsstufen hinweg. Hier ist eine Empowerment-orientierte Organisations- und Personalentwicklung erforderlich. Um diesem Entwicklungssprung von Mobile Learning Rechnung zu tragen, etabliert sich zunehmend der Begriff Seamless Learning bzw. Mobile Assisted Seamless Learning. Dieser Beitrag möchte daher einen umfassenden konzeptionellen Rahmen zur Einordnung und zum Verständnis von Mobile Learning im betrieblichen Bildungsmanagement liefern. Ziel ist es, Ausgangslage, Perspektiven sowie künftige Entwicklungslinien für den Einsatz von Mobile Learning in der betrieblichen Bildung aufzuzeigen.

\section{Schlüsselwörter}

Mobile Learning, Seamless Learning, Seamless Mobile Learning, Arbeitswelt 4.0, Empowerment-orientierte Organisationsentwicklung 


\section{$1 \quad$ Einleitung: Zielsetzung und Aufbau des Beitrages}

Mobile Learning ist schon seit gut zehn Jahren ein Thema (vgl. Meier 2005), hat sich aber in den vergangenen Jahren zu einem wichtigen Entwicklungsfeld im Bereich des technologieunterstützten Lernens entwickelt (Specht und Ebner 2011). Auch im Kontext der beruflichen Bildung befindet sich Mobile Learning seit dem Aufkommen von Smartphones, Tablet PCs und speziell entwickelten Applikationen (Mobile Apps) im Aufwind. Wie so häufig, hat diese Entwicklung ihren Ursprung in der Verfügbarkeit neuer Technologien (Hug 2010).

Über die Jahre hat sich dabei auch die Bedeutung von „mobilem Lernen“ verändert. War mit mobilem Lernen ursprünglich der Zugriff auf Inhalte über mobile Endgeräte gemeint, wurden später darunter auch ganz allgemein Lernaktivitäten von mobilen Lernenden verstanden (Specht und Ebner 2011). Mit aktuellen Entwicklungen wie Cloud Computing, Internet der Dinge, Mobile Connectivity, Augmented bzw. Virtual Reality, Big Data und Cognitive Computing, die häufig unter dem Begriff „Arbeitswelt 4.0“ subsumiert werden, erfährt Mobile Learning nicht nur eine weiter zunehmende Bedeutung, sondern steht auch im Kontext einer grundlegenden digitalen Transformation von Wirtschaft und Gesellschaft. So stellt sich für Bildungsorganisationen ganz konkret die Frage, in welchem Rahmen Kompetenzentwicklung in der Arbeitswelt 4.0 gefördert und unterstützt werden soll. Hier sind zwei Ebenen zu integrieren: zum einen die (formalen und informellen) Lern- bzw. Entwicklungsprozesse auf den Ebenen von Individuen und Teams, zum anderen die Gestaltung von lern- und entwicklungsförderlichen Rahmenbedingungen auf der Ebene der Gesamtorganisation. Die Autonomie und die Selbststeuerung der einzelnen Mitarbeitenden und Teams werden wichtiger und drängen starre Vorgaben zur Kompetenzentwicklung in den Hintergrund. „Empowerment“ betont dabei eine Orientierung auf Ressourcen und Stärken anstelle einer traditionellen Fokussierung auf Wissens- oder Leistungsdefizite (Seufert et al. 2017).

Neuere Ansätze im Bildungsmanagement sehen die Potenziale von Mobile Learning nicht nur in zeit- und ortsunabhängigem Lernen, in der Verbindung von informellem und formellem Lernen, sondern auch bei der Unterstützung von Lernen entlang einer Bildungsbiographie über verschiedene Bildungsstufen hinweg. Auch hier ist eine Empowerment-orientierte Organisations- und Personalentwicklung erforderlich. In diesem Sinne wird eine Aufteilung in Aus- und Weiterbildung immer mehr obsolet - es geht um Kompetenzentwicklung und lebenslanges Lernen. Um diesem Entwicklungssprung von Mobile Learning Rechnung zu tragen, etabliert sich zunehmend der Begriff Seamless Learning bzw. Seamless Mobile Learning (Wong 2012; Wong et al. 2015).

Dieser Beitrag möchte daher einen umfassenden konzeptionellen Rahmen zur Einordnung und zum Verständnis von Mobile Learning im betrieblichen Bildungsmanagement liefern. Ziel ist es, Ausgangslage, Perspektiven sowie Entwicklungslinien für den Einsatz von Mobile Learning in der betrieblichen Bildung aufzuzeigen. Der Beitrag ist daher nach folgenden Leitfragen gegliedert: 
1. Nutzung: Wie ist Mobile Learning derzeit in der betrieblichen Bildung verbreitet und wie wird die zukünftige Nutzung eingeschätzt?

2. Potenziale und Hürden: Welche Erkenntnisse gibt es hinsichtlich der Potenziale, aber auch der Hürden beim Einsatz von Mobile Learning in der betrieblichen Bildung?

3. Perspektiven: Welche (unterschiedlichen) Perspektiven für Mobile Learning in der betrieblichen Bildung zeigen sich?

4. Entwicklungslinien im Kontext Arbeitswelt 4.0: Wie müssen künftig Maßnahmen zur Kompetenzentwicklung in einer Arbeitswelt 4.0 gestaltet sein und welche Bedeutung erhält Mobile Learning in diesem Zusammenhang?

Den Abschluss des Beitrages bilden ein kurzes Fazit sowie ein Ausblick auf künftige Forschungsfragen für die Weiterentwicklung von Mobile Learning im betrieblichen Bildungsmanagement.

\section{$2 \quad$ Aktueller Stand und zukünftige Nutzung von Mobile Learning in Unternehmen}

\subsection{Aktueller Stand}

Wie die Benchmarking-Studie 2015 des eLearning Journals (vgl. eLearning Journal 2015) zeigt, spielte Mobile Learning zum Zeitpunkt der Befragung Ende 2014 für 61 \% der Unternehmen für das formale Training noch keine Rolle. Weitere 20,2 \% der Studienteilnehmer und -teilnehmerinnen gaben an, dass mobile Endgeräte nur selten zum Einsatz kommen. Immerhin in $13 \%$ der Unternehmen ist Mobile Learning gelegentlich ein Thema. Relativ selten sind dagegen Unternehmen, die oft $(4,6 \%)$ oder sogar immer $(1,1 \%)$ Mobile Learning im Rahmen der internen Aus- und Weiterbildung anbieten. Damit liegt der Einsatz von mobilen Endgeräten in der betrieblichen Bildung sogar knapp hinter anderen Trend-Themen, wie beispielsweise Social Learning oder Micro Learning, und deutlich hinter den etablierten E-Learning-Formaten wie WBTs, Blended Learning oder auch virtuelle Klassenräume. Lediglich MOOCs und Game Based Learning werden in deutschsprachigen Unternehmen aktuell noch seltener genutzt (eLearning Journal 2015). 


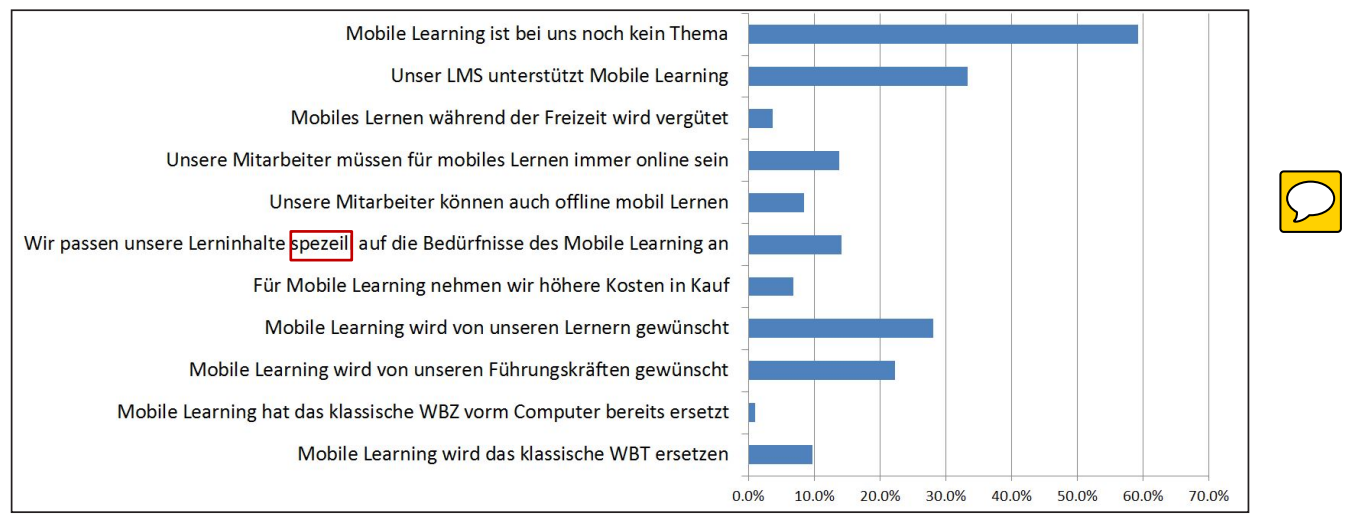

Abb. 1 Bestandsaufnahme zu Mobile Learning (eLearning Journal 2015; eigene Darstellung)

Auch eine Delphi-Studie des MMB-Instituts und der Haufe Akademie mit Beteiligung von Experten aus KMU sowie aus Großunternehmen zeigt, dass die aktuelle - aber auch die für die unmittelbare Zukunft erwartete - Nutzung von mobilem Lernen weiter hinter anderen Formaten, wie z.B. WBT oder virtuelle Klassenzimmer, zurücksteht (Michel 2014). Dabei zeigte sich, dass mobile Lernanwendungen mit insgesamt $38 \%$ (aktuelle + künftige Nutzung in KMU) bzw. 53 \% (aktuelle + künftige Nutzung in Großunternehmen) zurückstehen (vgl. Abbildung 2).

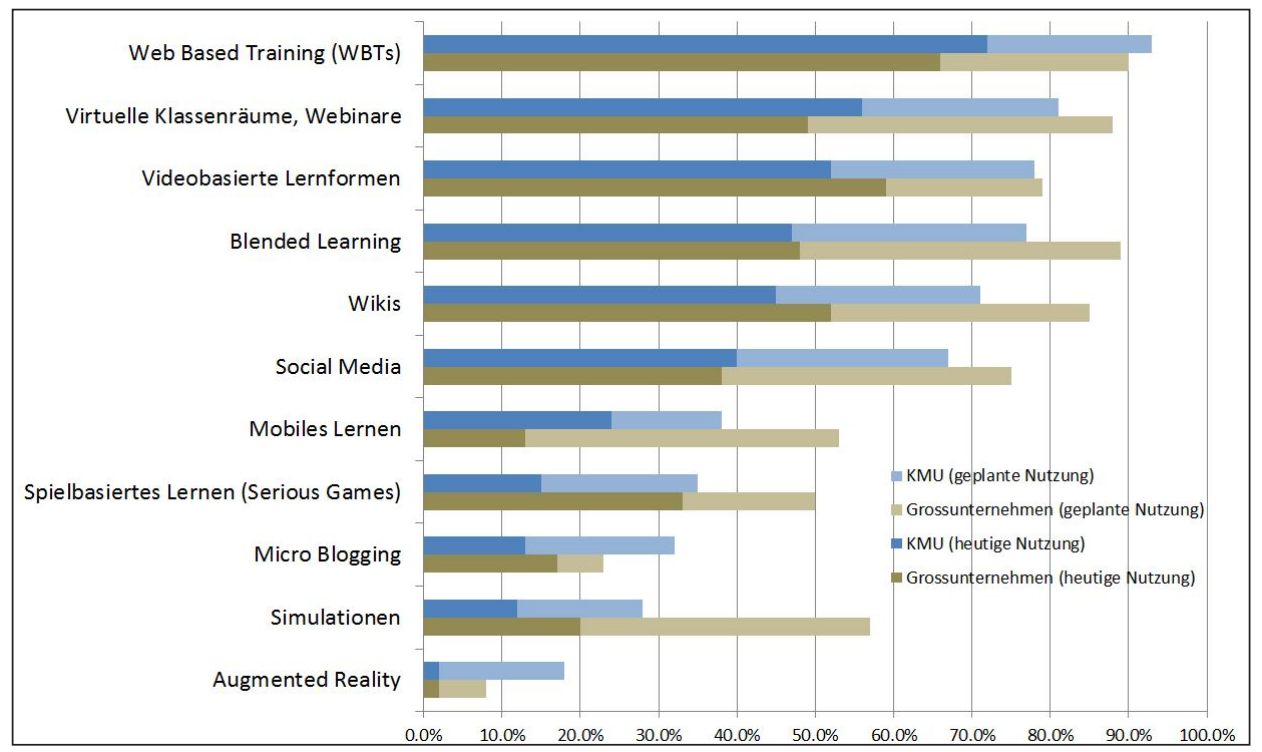

Abb. 2 Aktuelle und zukünftige Nutzung verschiedener Lernformen (Michel 2014; eigene Darstellung) 


\subsection{Zukünftige Bedeutung von Mobile Learning in Unternehmen}

Eine aktuelle Studie des MMB-Instituts zeigt aber, dass unter verschiedenen technologischen Neuerungen (Analytics, adaptive und intelligente Systeme, Big Data, Virtual Reality etc.) für die nächsten zehn Jahre die größte Bedeutung bei mobilem Lernen gesehen wird (MMB Institut 2016 und Abbildung 3).

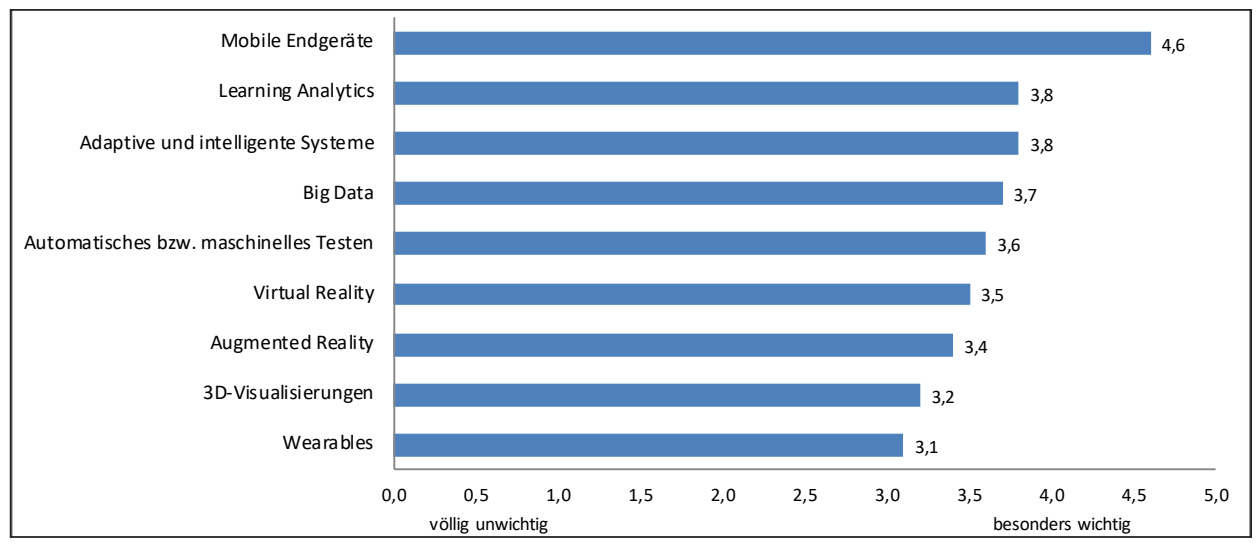

Abb. 3 Technologische Trends in der digitalen Bildung (MMB Institut 2016; eigene Darstellung)

Die befragten Expertinnen und Experten wurden darüber hinaus gefragt, welche Argumente ihrer Einschätzung zugrunde liegen. Mobile Endgeräte - in der Regel verstanden als Smartphones, nicht Tablets oder Laptops - werden in erster Linie wegen ihrer ubiquitären Verfügbarkeit und ihrer großen Verbreitung in allen Bevölkerungsschichten als dominantes Lernwerkzeug der Zukunft gesehen. Hinzu komme, dass „jeder damit umgehen kann“. Hier wird von einigen Befragten auf die Generation der „Digital Natives“ verwiesen, „für die der Umgang mit mobilen Endgeräten selbstverständlich ist“. Wegen der großen Verbreitung von Smartphones biete sich im Übrigen das Konzept des BYOD („Bring your own device“) an, das die Kosten für Bildungsträger merklich begrenze. Der Hinweis auf die zunehmende Konvergenz der Endgeräte wird ebenfalls zur Unterstützung für diesen Trend angeführt: „Alles wird mobil.“ Damit würden Smartphones und Tablets zur „wichtigsten Schnittstelle zum digitalen Ökosystem“ (MMB Institut 2016).

Allerdings spricht sich eine Minderheit von etwa $20 \%$ der Experten und Expertinnen gegen die These aus, dass mobile Endgeräte der wichtigste technologische Zukunftstrend sind. Ihre Argumente beziehen sich auf pädagogische Restriktionen ebenso wie auf technische Entwicklungen. Mit ihrer Konzentration auf das „Lernen in kleinen Portionen“ seien mobile Geräte nur für einen begrenzten Bereich von Lerngegenständen geeignet. Auch fehle „trotz intensiver Forschungsarbeit“ der Nachweis ihrer generellen Eignung als Lernmittel. 
Ein wichtiges Argument gegen die Einschätzung mobiler Endgeräte als „technologischer Zukunftstrend Nr. 1“ ist darüber hinaus die begrenzte Haltbarkeit von Zukunftsprognosen für digitale Tools und Anwendungen generell. Einer der befragten Experten formuliert das so: „Es könnte in den kommenden Jahren neue technische Möglichkeiten geben, die wir heute noch nicht einmal erahnen." (MMB Institut 2016)

Während also die Zahlen zur aktuellen Verbreitung von mobilem Lernen eher ernüchternd sind, sind die Erwartungen für Mobile Learning in den kommenden Jahren weiterhin recht positiv. Es bleibt daher abzuwarten, ob sich die Nutzung den Erwartungen in der nächsten Zeit tatsächlich annähern wird.

\section{Treiber und Barrieren für den Einsatz von Mobile Learning in Unternehmen}

\subsection{Erwartungen zum zukünftigen Einsatz von Mobile Learning}

Ein Grund für die bisher eher verhaltene Umsetzung von mobilem Lernen liegt vermutlich darin, dass vielen Entscheidungsträgern Wissen dazu fehlt, welche Möglichkeiten mobiles Lernen bietet und wie es umgesetzt werden kann. In einer Studie zur Akzeptanz von mobilem Lernen schälen Beutner und Pechuel verschiedene akzeptanzförderliche Faktoren (Vorteile) und Akzeptanzbarrieren (Nachteile) von mobilem Lernen sowie gleichzeitig Erwartungen an diese Modalität heraus (Beutner und Pechuel 2012). Abbildung 4 führt die dabei genannten Aspekte - getrennt nach Kategorien bzw. Säulen - auf. Dabei sind die von den befragten Entscheidungsträgern am häufigsten genannten Aspekte oben aufgeführt, die am wenigsten häufig genannten Aspekte unten.

Das Bild, das sich hierbei ergibt - Flexibilität und (erwartete) Kostenreduktionen sind wichtige Treiber für die Umsetzung von mobilem Lernen -, wird durch eine systematische Analyse weiterer Studien bestätigt (vgl. Tabelle 1): 


\begin{tabular}{|l|l|}
\hline $\begin{array}{l}\text { Uöglichkeit, schnell an } \\
\text { Informationen zu gelangen }\end{array}$ \\
\hline $\begin{array}{l}\text { Wlexibilität } \\
\text { genutzeit werden }\end{array}$ \\
\hline $\begin{array}{l}\text { Möglichkeit, schnell Informationen } \\
\text { und Wissen zu teilen }\end{array}$
\end{tabular}

Abb.4 Einschätzung von Entscheidungsträgern bezüglich der Akzeptanz von Mobile Learning

Tab. 5 Vorteile von Mobile Learning (eigene Untersuchung/Darstellung)

Flexibilität (Zeit und Ort)

Kosten geringer

Individualisiertes Lernen

Wartezeiten können sinnvoll genutzt werden

Qualitätsverbesserung des Lernens

Entlastung des Aus- und Weiterbildungspersonals

schneller/ständiger Zugang zu Daten/Informationen

fördert selbstgesteuertes Lernen

Lernerfolgskontrolle

Kombination verschiedener Medien- und Präsentationsformen

Motivationssteigerung

Unterstützung heutiger oder zukünftiger Unternehmensstrategien

Unterstützung der Personalentwicklung

Entwicklung/Bildung virtueller Kompetenzteams

Betreuungsaufwand geringer

Smartphones sind nahe am Alltag von jungen Menschen

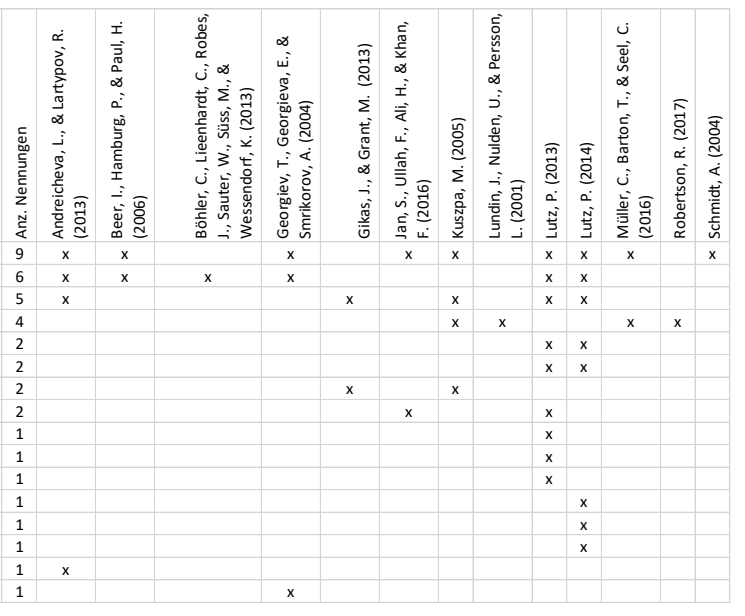




\subsection{Hürden für eine zunehmende Verbreitung von Mobile Learning}

Angesichts der potenziellen Vorteile von Mobile Learning stellt sich die Frage, warum die Umsetzung von mobilem Lernen in Unternehmen nicht schon weiter vorangeschritten ist. Hier spielen die (erwarteten) hohen Anschaffungskosten, die mit dem Aufbau einer Mobile Learning-Infrastruktur verbunden sind (Anschaffung von Smartphones und Tablets) eine wichtige Rolle. Die hohen Anschaffungskosten für die mobilen Endgeräte ließen sich zwar durch einen BYOD-Ansatz vermeiden (BYOD = Bring Your Own Device), wobei dabei Fragen der Datensicherheit berücksichtigt werden müssen (BYOD = Bring Your Own Disaster). Eine weitere wichtige Barriere sind die (unzureichenden) medientechnischen Fertigkeiten der Mitarbeitenden (vgl. Abbildung 4 sowie Tabelle 2). Darüber hinaus werden auch technische Hürden (kleine Bildschirme) angeführt (Tabelle 2). Schließlich spielen auch Herausforderungen im Hinblick auf die bereits etablierte Infrastruktur für E-Learning eine Rolle.

Hier stellen etablierte Lern-Management-Systeme eine Barriere dar. So zeigt die eLearning BENCHMARKING Studie 2015, dass lediglich bei 33,2 \% der befragten Teilnehmenden das eigene LMS bereits Mobile Learning unterstützt.

Tab. 6 Nachteile von Mobile Learning (eigene Untersuchung/Darstellung)

\begin{tabular}{|l|}
\hline kleiner Bildschirm \\
\hline hohe Selbstlernkompetenz notwendig \\
\hline persönlicher Kontakt/Austausch fehlt/ist eingeschränkt \\
\hline Umstellung fällt den Lernenden schwer \\
\hline Eingabe über Tastatur ist umständlich \\
\hline technische Grenzen/Fehler \\
\hline Ablenkung durch die Geräte \\
\hline limitierte Speicherkapazität \\
\hline Normierung auf unterschiedliche Geräte aufwändig \\
\hline fehlende Regelungen zum Lernen am Arbeitsplatz \\
\hline mangelnde Unterstützung durch Vorgesetzte \\
\hline Intrasparenz des E-Learning Marktes \\
\hline mangelhaftes Marketing für E-Learning \\
\hline fehlende Abschlüsse/Zertifikate \\
\hline Prüfung des Lernerfolges schwierig \\
\hline hohe Anfangsinvestition \\
\hline häufige Störungen im Lernprozess \\
\hline guter Umgang mit Technologien notwendig \\
\hline Entwicklung teuer/aufwändig \\
\hline Akkus halten nicht lange \\
\hline Kosten für Internetnutzung \\
\hline
\end{tabular}

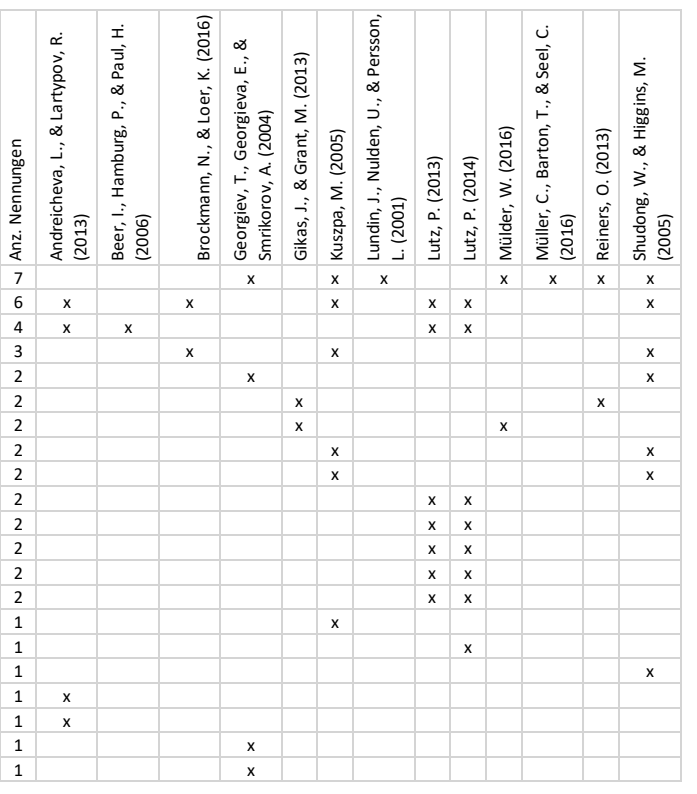

In einigen Studien (siehe den Überblick in Tabelle 3) werden darüber hinaus Innovationsbarrieren betont, die oft in den erforderlichen Voraussetzungen begründet sind. Genannt 
werden hier unter anderem die Lernkultur, einfacher technischer Zugang sowie auch Fragen zur Auswertung/Nutzung der Datenspuren, die bei mobilem Training entstehen.

Tab. 7 Voraussetzungen von Mobile Learning (eigene Untersuchung/Darstellung)

Lernförderliche Unternehmenskultur

\begin{tabular}{|c|c|c|c|c|}
\hline 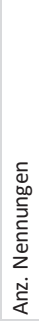 & 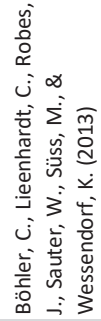 & 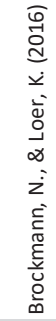 & 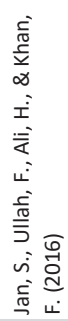 & 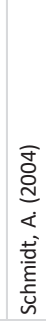 \\
\hline 1 & $\mathrm{x}$ & & & \\
\hline 1 & & $x$ & & \\
\hline 1 & & & $x$ & \\
\hline 1 & & & & $x$ \\
\hline
\end{tabular}

Good Practice-Beispiele im Hinblick auf mobiles Lernen beinhalten somit auch einen umfassenderen Ansatz, der über technische Fragen hinausgeht und auch Aspekte wie adressierte Zielgruppen, Zielsetzungen für mobiles Lernen, konkrete Lernziele und Aktivitäten, Benutzeroberflächen, Kanäle und Kanalintegration, Netzwerk und Zugriffsberechtigungen sowie Endgeräte einschließt (vgl. Abbildung 5). Dabei ist wichtig, dass diese Gestaltungsaspekte nicht nur einmal definiert, sondern kontinuierlich wechselseitig in Passung zueinander gebracht werden.
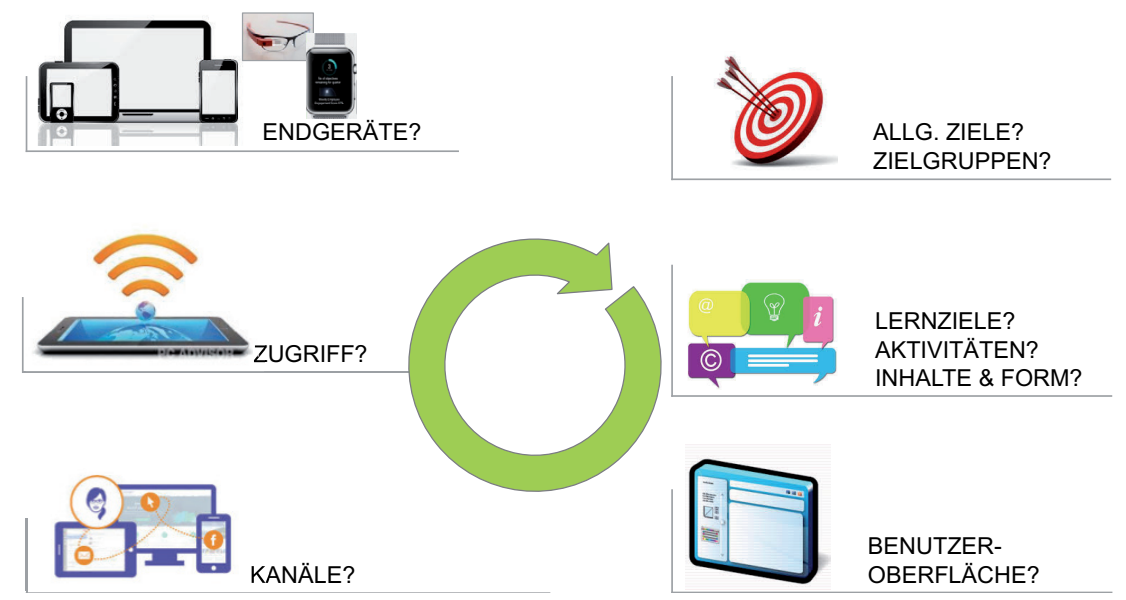

Abb.5 Gestaltungsaspekte von mobilem Lernen (eigene Darstellung nach Wentworth 2014) 


\section{$4 \quad$ Perspektiven auf Mobile Learning}

\subsection{Von einer technischen zu einer didaktischen Perspektive auf Mobile Learning}

Mit Mobile Computing vereinfacht sich für die mobil Lernenden der Zugriff auf die Inhaltsfülle des World Wide Web, und sie können an jedem Ort und zu jeder Zeit in das „soziale“ Web 2.0 eintauchen (z. B. Kremer und Pferdt 2008). Für die berufliche Ausbildung bietet Mobile Learning u.a. Potenziale zur Verbesserung der Lernortkooperation. So können beispielsweise Tablets lernortunabhängig eingesetzt werden, um situationsgerecht auf Lerninhalte zuzugreifen oder auch, um Lernerfahrungen handlungsnah zu dokumentieren. Allerdings geht es beim Thema mobiles Lernen aus unserer Sicht weniger um bestimmte Zugriffsmodalitäten auf Lerninhalte bzw. digitale Lernumgebungen, sondern mehr um die pädagogisch-didaktische Ausgestaltung von Lehr-Lernszenarien.

Hier bietet sich der Rückgriff auf etablierte (wirtschafts-) didaktische Ordnungsraster zu Lehr-Lernmethoden an, beispielsweise nach Euler und Hahn (2014: 295). Euler und Hahn kombinieren bei ihrem Ordnungsraster jeweils eine Aktions- und eine Sozialform des Lernens bzw. Lehrens. So ergibt sich ein Lehrvortrag bzw. eine Vorführung aus der Kombination der Aktionsform „Darbieten“ mit der Sozialform „Plenum“. Die verschiedenen Sozial- und Aktionsformen werden entsprechend zugrunde gelegter Lernziele, die mit einer didaktischen Methode erreicht werden sollen, ausgewählt. Analog zu dieser Struktur lassen sich auch für das Mobile Learning Szenarien als eine Kombination von Sozial- und Aktionsform entwerfen, wobei jedoch die Besonderheiten mobiler Endgeräte und mobiler Lernprozesse berücksichtigt werden müssen. So findet Mobile Learning häufig außerhalb formaler (schulischer) Lernsettings statt, also z. B. Zuhause oder am Ausbildungsplatz. Dementsprechend wird die Aktionsform nicht als Interaktion zwischen dem Lehrenden und den Lernenden definiert, wie im klassischen Präsenzunterricht. Vielmehr bezeichnet die Aktionsform beim Mobile Learning die Aktivität der Lernenden in der Auseinandersetzung mit dem mobilen Endgerät. Denn je nach Aktivität moderiert das mobile Endgerät die pädagogischen Interaktionen der Lehrenden mit Inhalten (in Form eines E-Books), mit anderen Lernenden (z. B. über ein Online Social Network) oder mit Lehrenden/Ausbildenden. Auf der Aktivitätsdimension werden drei zentrale Aktivitätsformen unterschieden, die sich an bekannten Lernzieltaxonomien orientieren (vgl. Anderson und Krathwohl 2001):

- Inhalte aneignen: Die Lernenden befassen sich mit Inhalten, die in unterschiedlichen Modalitäten (Text, Pod-/Videocast etc.) über das mobile Endgerät abrufbar sind.

- Problemstellungen dokumentieren und bearbeiten: Die Lernenden bearbeiten Problemstellungen in unterschiedlichen Kontexten (Schule, Betrieb; formal, informell) mit Hilfe mobiler Endgeräte (z. B. auf Basis von Fotos oder Videos) individuell oder in der Diskussion mit anderen.

- Erfahrungen dokumentieren, reflektieren und weitergeben: Die Lernenden erstellen eigene Medienprodukte, die etwa der Dokumentation von Lernerfahrungen bzw. Pro- 
blemlösungen dienen. Denkbar ist hier z. B. ein Rollenspiel, in dessen Rahmen eine erfolgreiche Strategie zur Bewältigung kritischer Kundengespräche dargestellt wird.

Auch die Sozialformen können entsprechend der Möglichkeiten, die Mobile Computing bzw. Mobile Learning bietet, spezifiziert werden. Hier spielen die Möglichkeiten der virtuellen Vernetzung eine wichtige Rolle. So können Lernende auch außerhalb des Klassenraums bzw. des Ausbildungsortes mit Kolleginnen und Kollegen in Kontakt treten und sich austauschen. Folgende Sozialformen müssen daher berücksichtigt werden:

- Individuelles Lernen;

- Lernen in der Gruppe, z. B. in einer Kleingruppe innerhalb des Klassenverbands, Lernpartnerschaften am Arbeitsplatz;

- Lernen in lernortübergreifenden Communities, z. B. virtuelle Communities of Practice (Lave und Wenger 1991), die sich informell in Online Social Networks organisieren.

Abbildung 6 illustriert ein Beispiel für die didaktische Landkarte von Mobile Learning, deren Breitengrade die Sozialformen und deren Längengrade die Lerneraktivitäten darstellen. Innerhalb dieses Koordinatensystems können verschiedene Lehr-Lernszenarien verortet werden. Die Darstellung als unterschiedliche „Inseln“ verweist darauf, dass die

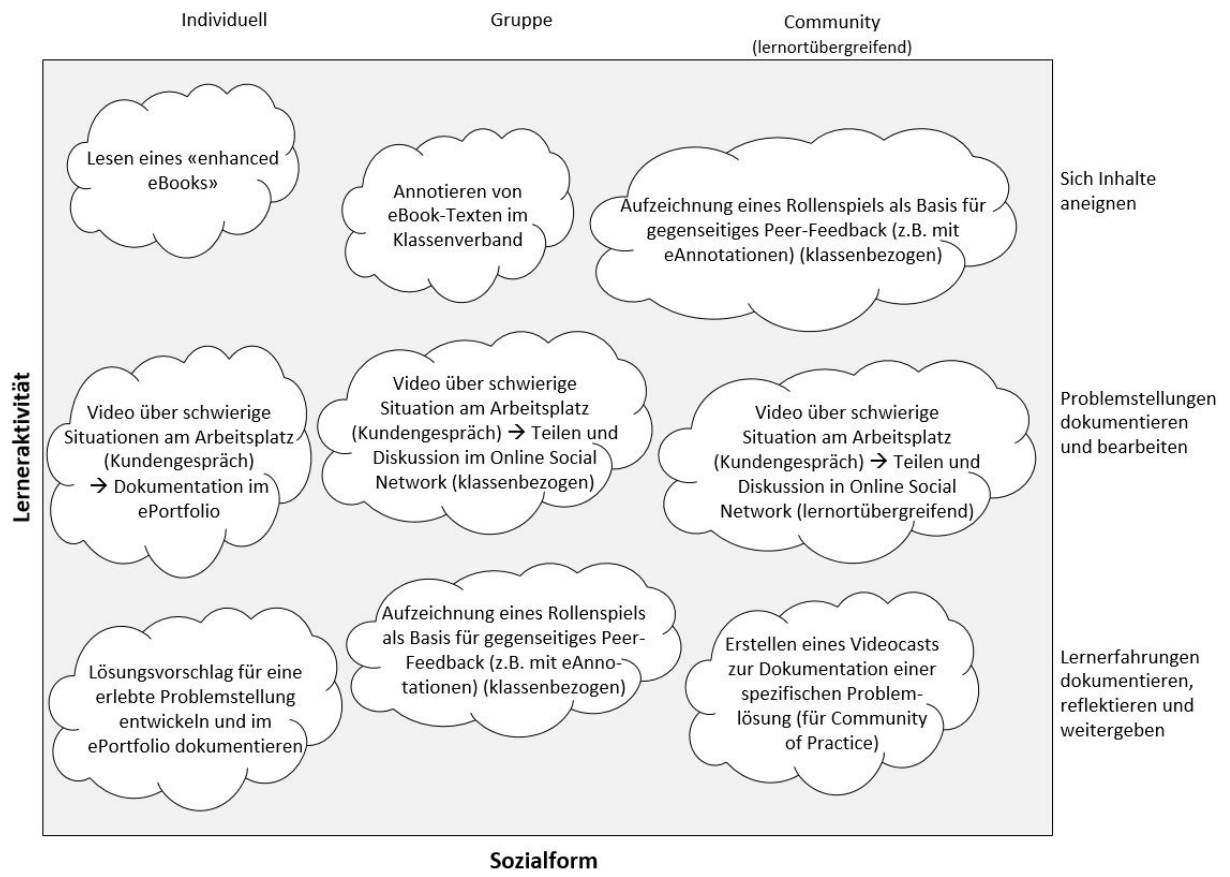

Abb. 6 Landkarte zur Verortung von Mobile Learning-Szenarien mit Tablet PCs 
Übergänge zwischen den einzelnen Szenarien fließend sind und das Schema nicht auf eine starre Einteilung zielt, sondern eine heuristische Hilfestellung anbieten will.

Eine Sichtung verschiedener Studien zur Umsetzung von mobilem Lernen zeigt, dass die dargestellten Projekte sowohl technische als auch didaktische Zielsetzungen verfolgen. Darüber hinaus wird deutlich, dass Schulen eher didaktische Überlegungen mit einbeziehen während Unternehmen eher auf die technische Umsetzung fokussieren. Eine Bewegung von eher technisch zu eher didaktisch ausgerichteten Projekten über die Jahre kann allerdings nicht eindeutig festgestellt werden.

Tab. 8 Übersicht zu Studien zu mobilem Lernen (eigene Untersuchung/Darstellung)

\begin{tabular}{|c|c|c|c|c|}
\hline $\begin{array}{l}\text { Autoren/ } \\
\text { Autorinnen }\end{array}$ & Jahr & Mobile Learning-Einsatz & Bereich \& Land & $\begin{array}{l}\text { Fokus } \\
\text { (didaktisch, technisch) }\end{array}$ \\
\hline Robertson & 2017 & $\begin{array}{l}\text { Fortbildung } \\
\text { PharmaKonzern }\end{array}$ & $\begin{array}{l}\text { Unternehmen, } \\
\text { Kenia }\end{array}$ & $\begin{array}{l}\text { Technisch } \\
\text { (App entwickeln) }\end{array}$ \\
\hline $\begin{array}{l}\text { Brockmann \& } \\
\text { Loer }\end{array}$ & 2016 & $\begin{array}{l}\text { Online-Seminar } \\
\text { Bachelor-Kurs }\end{array}$ & $\begin{array}{l}\text { Schule, } \\
\text { Deutschland }\end{array}$ & $\begin{array}{l}\text { Didaktisch (Aktive Mit- } \\
\text { arbeit fördern, Theorie } \\
\text { und Praxis verbinden) }\end{array}$ \\
\hline $\begin{array}{l}\text { Müller, Stahl, } \\
\text { Lübcke \& } \\
\text { Alder }\end{array}$ & 2016 & $\begin{array}{l}\text { FLEX Studiengang } \\
\text { ZHAW }\end{array}$ & Schule, Schweiz & $\begin{array}{l}\text { Didaktisch (flexibles, } \\
\text { selbstbestimmtes Lernen } \\
\text { fördern) }\end{array}$ \\
\hline $\begin{array}{l}\text { Jan, Ullah, } \\
\text { Ali \& Khan }\end{array}$ & 2016 & $\begin{array}{l}\text { Einsatz von mobilen } \\
\text { Geräten an Universitäten }\end{array}$ & Schule, Pakistan & $\begin{array}{l}\text { Technisch \& didaktisch } \\
\text { (technische Umsetzung } \\
\text { und Lernoutput) }\end{array}$ \\
\hline $\begin{array}{l}\text { Decker, } \\
\text { Wesseloh \& } \\
\text { Schumann }\end{array}$ & 2015 & Mobile Lernanwendung & $\begin{array}{l}\text { Unternehmen, } \\
\text { Deutschland }\end{array}$ & $\begin{array}{l}\text { Didaktisch (Steigerung } \\
\text { der Motivation) }\end{array}$ \\
\hline $\begin{array}{l}\text { Harriehausen- } \\
\text { Mühlbauer \& } \\
\text { Sonne }\end{array}$ & 2013 & Lernapp QuizLounge & $\begin{array}{l}\text { Unternehmen, } \\
\text { Deutschland }\end{array}$ & $\begin{array}{l}\text { Technisch } \\
\text { (App entwickeln) }\end{array}$ \\
\hline de Witt & 2012 & $\begin{array}{l}\text { Mobile Lernanwendung } \\
\text { für Berufskraftfahrer }\end{array}$ & $\begin{array}{l}\text { Unternehmen, } \\
\text { Deutschland }\end{array}$ & $\begin{array}{l}\text { Didaktisch (zielgruppen- } \\
\text { gerechte Didaktik) }\end{array}$ \\
\hline
\end{tabular}

\subsection{Von einer Methoden- zu einer Zielinnovation des Mobile Learning}

Didaktische Innovationen können auf den Einsatz neuer Technologien zum Erreichen bisheriger Ziele, auf die Umsetzung neuer Methoden oder aber auch auf das Erreichen neuer Entwicklungsziele ausgerichtet sein (vgl. Seufert 2013). Im Hinblick auf den zuletzt genannten Aspekt ist hervorzuheben, dass mobiles Lernen Potenzial insbesondere für die Entwicklung von digitalen Kompetenzen (bzw. Kompetenzen für eine digitale Lebens- und Arbeitswelt) bietet. Im Hinblick auf die Förderung von medienbezogenen Handlungskompetenzen erscheinen besonders drei Bereiche zentral: 
- die Förderung von arbeitsbezogener Handlungskompetenz (Euler und Hahn 2014),

- die Förderung von Selbstlernkompetenzen (Friedrich und Mandl 1997) sowie

- die Förderung von Medienkompetenzen (Aufenanger 2001).

Die nachfolgende Tabelle gibt anhand des Fallbeispiels CYP (Center for Young Professionals; vgl. Seufert et al. 2012) einen Überblick über die drei Kompetenzbereiche sowie die jeweils identifizierten Potenziale des Einsatzes von mobilen Endgeräten, wie beispielsweise Tablets. Dies illustriert, dass mit dem Tablet-Einsatz tatsächlich vorwiegend Ziele angesprochen werden können, die über das bisherige Zielspektrum hinausgehen und im Rahmen bislang etablierter Lernumgebungen schwierig zu fördern sind.

Tab.9 Potenziale des Tablet-Einsatzes zur Förderung medienbezogener Handlungskompetenzen im Rahmen des Fallbeispiels CYP

\begin{tabular}{|c|c|c|}
\hline $\begin{array}{l}\text { Kompetenz- } \\
\text { bereiche }\end{array}$ & Schwerpunkte Fallbeispiel CYP & Mehrwert Tablet-Einsatz \\
\hline $\begin{array}{l}\text { Arbeitsbezogene } \\
\text { Handlungs- } \\
\text { kompetenzen }\end{array}$ & $\begin{array}{l}\text { Arbeit mit digitalen Technologien } \\
\text { im Bankenumfeld (Stichwort } \\
\text { E-Banking). Veränderungen } \\
\text { durch den Tablet PC aufnehmen. }\end{array}$ & $\begin{array}{l}\text { - „Banking 2.0“: Tablet PC als künf- } \\
\text { tiges Beratungsinstrument wird an } \\
\text { Bedeutung gewinnen. } \\
\text { - Multimodaler und vernetzter } \\
\text { Zugang zu Lerninhalten. }\end{array}$ \\
\hline \multirow[t]{2}{*}{$\begin{array}{l}\text { Selbstlern- } \\
\text { kompetenzen }\end{array}$} & $\begin{array}{l}\text { In Lerntandems und Lerngrup- } \\
\text { pen Fallsituationen bearbeiten: } \\
\text { Bei der Bearbeitung von Fällen } \\
\text { Feedback geben. Sich zu au- } \\
\text { thentischen Fallsituationen am } \\
\text { Arbeitsplatz austauschen. Eigene } \\
\text { Fallsituationen generieren. }\end{array}$ & $\begin{array}{l}\text { Mobiler Zugang und vereinfachte } \\
\text { Kommunikation im Austausch mit } \\
\text { anderen Auszubildenden. } \\
\text { - Videofunktionalitäten ermögli- } \\
\text { chen eine intensive Auseinander- } \\
\text { setzung mit Beratungssituationen } \\
\text { sowie das Annotieren von Videos } \\
\text { in Gruppen (z.B. Kommentierung } \\
\text { aus unterschiedlichen Perspek- } \\
\text { tiven, fokussiert nach didaktisch } \\
\text { begründeten Kriterien). }\end{array}$ \\
\hline & $\begin{array}{l}\text { Selbststeuerung des Lernrah- } \\
\text { mens: Lernziele selbst bestim- } \\
\text { men. Lernfortschritte überprü- } \\
\text { fen (z. B. Ausbau des Bereichs } \\
\text { „Invididual Learning“ im zweiten } \\
\text { Lehrjahr). }\end{array}$ & $\begin{array}{l}\text { - Unterstützung beim Erstellen/ } \\
\text { Aktualisieren von eigenen Lern-/ } \\
\text { Zeitplänen sowie erleichterter } \\
\text { Austausch dazu, z. B. Abgleich } \\
\text { von Lernplänen mit den anderen } \\
\text { Auszubildenden. } \\
\text { - Nutzung von Annotationsfunk- } \\
\text { tionen bei der Bearbeitung von } \\
\text { Materialien. Erstellen persönlicher } \\
\text { Mindmaps oder Wissensland- } \\
\text { karten. }\end{array}$ \\
\hline
\end{tabular}




\begin{tabular}{|c|c|c|}
\hline \multirow{3}{*}{$\begin{array}{l}\text { Kompetenz- } \\
\text { bereiche }\end{array}$} & Schwerpunkte Fallbeispiel CYP & Mehrwert Tablet-Einsatz \\
\hline & $\begin{array}{l}\text { Transferstrategien: Prob- } \\
\text { lemlösungen nachvollziehen. } \\
\text { Übertragung in andere Kontexte } \\
\text { vornehmen (Transferaufträge im } \\
\text { Arbeitskontext dokumentieren } \\
\text { und kommentieren). }\end{array}$ & $\begin{array}{l}\text { Mediale Aufnahme/Dokumenta- } \\
\text { tionen von Transferaufträgen vor } \\
\text { Ort (z. B. in Form von Bildern, } \\
\text { Videos). Brückenschlag zwischen } \\
\text { verschiedenen Lernorten, z. B. } \\
\text { Schule und Arbeitsplatz in der } \\
\text { Bank. }\end{array}$ \\
\hline & $\begin{array}{l}\text { Metakognition: Reflexionspro- } \\
\text { zesse über das eigene Lernen } \\
\text { unterstützen (insbes. eigene Lern- } \\
\text { voraussetzungen kritisch über- } \\
\text { prüfen und ggf. modifizieren). }\end{array}$ & $\begin{array}{l}\text { Mobiler Zugriff auf das Lernjour- } \\
\text { nal. Erweiterte Strukturierungs- } \\
\text { möglichkeiten für das Lernjournal } \\
\text { jenseits einer sequentiellen Abfolge } \\
\text { von Einträgen. Erinnerungsfunk- } \\
\text { tionen und vom System generierte } \\
\text { Aufforderungen zur Reflexion. }\end{array}$ \\
\hline $\begin{array}{l}\text { Medien- } \\
\text { kompetenzen }\end{array}$ & $\begin{array}{l}\text { Informationsbeschaffungs- und } \\
\text {-verarbeitungsstrategien: Darge- } \\
\text { botene Informationen aufnehmen } \\
\text { und strukturieren (Notizen ma- } \\
\text { chen). Informationen beschaffen, } \\
\text { ordnen und beurteilen. Wesentli- } \\
\text { ches und Unwesentliches trennen. } \\
\text { Medienkritik: Informationen } \\
\text { verschiedener Quellen beurtei- } \\
\text { len. Angemessenen Umgang mit } \\
\text { Privatheit und Informations- } \\
\text { sicherheit praktizieren. }\end{array}$ & $\begin{array}{l}\text { Tablet-PC vereinfacht Suche nach } \\
\text { Information und die Einbindung } \\
\text { tagesaktueller Informationen in } \\
\text { der Präsenzlehre. } \\
\text { - Nutzung von Annotationsfunk- } \\
\text { tionen für persönliche Notizen } \\
\text { sowie von Strukturierungshilfen } \\
\text { zur Organisation von Informati- } \\
\text { onen (z.B. Social Bookmarking, } \\
\text { Tagging-Funktionalitäten). }\end{array}$ \\
\hline
\end{tabular}

\section{$5 \quad$ Künftige Entwicklungslinien für Mobile Learning in der Arbeitswelt 4.0}

\subsection{Arbeitswelt 4.0}

Unsere Gesellschaft unterliegt derzeit tiefgreifenden und dynamischen Veränderungen durch soziokulturelle, technologische und demographische Entwicklungen wie z.B. Globalisierung, Wertewandel, Fachkräftemangel und eine zunehmende Digitalisierung (Behrmann 2006; Schuchmann und Seufert 2015; Seufert 2013).

Im Rahmen des gemeinsam von Shareground und der Universität St. Gallen, Institut für Medien und Kommunikationsmanagement, erstellten Studie „Arbeit 4.0: Megatrends digitaler Arbeit der Zukunft“ (Shareground 2015) wurden eine Reihe von übergreifenden Trends im Hinblick auf die Arbeitswelt von morgen identifiziert. Diese Trends wurden 
den Bereichen „Organisation“, „Arbeit“ und „Führung“ zugewiesen, und sie beinhalten u. a. folgende Aussagen:

- Peer-to-peer-Zusammenarbeit verdrängt Hierarchien;

- vertraglich vereinbarte Arbeitseinsätze statt Arbeitsverhältnisse (Beauftragen statt Einstellen);

- Arbeit wird entgrenzt, die räumliche Verortung von Leistungserbringern nebensächlich;

- Mitarbeitende werden zunehmend über „Brot und Spiele“ gebunden;

- Selbstmanagement wird zu einer zentralen Kernkompetenz;

- Beidhändigkeit („explore new business AND exploit current business“) wird zentral.

Diese Trends und die diesbezüglich erwarteten Veränderungen haben Implikationen für das Personalmanagement und die Personalentwicklung von heute.

\subsection{Mobile Assisted Seamless Learning als integrierendes Konzept der Kompetenzentwicklung}

Vor dem Hintergrund der oben geschilderten Trends und Entwicklungen bietet das Konzept eines „Mobile Assisted Seamless Learning“ (siehe dazu auch den Beitrag von Ebner und Schön in diesem Handbuch) Potenzial für eine zukunfts- und Empowerment-orientierte Ausrichtung von Personal- und Kompetenzentwicklung. „Mobile Assisted Seamless Learning“ wird dabei wie folgt definiert als „a learning model where a student can learn whenever they are curious in a variety of scenarios and in which they can switch from one scenario or context (such as formal and informal learning, personal and social learning, etc.) to another easily and quickly using the personal device as a mediator" (Wong und Looi 2011: 1). In dieser Definition wird die Bedeutung von persönlichen Mobilgeräten (und implizit damit verbunden: mobilen, webbasierten Datenservices) als situationsübergreifende Lern-Infrastruktur hervorgehoben. Persönliche Mobilgeräte begleiten einen über verschiedene Kontexte (Zuhause, Unterwegs, am Arbeitsplatz) und ermöglichen damit eine anders kaum denkbare Nahtlosigkeit von Lernaktivitäten.

Wong und Looi haben im Rahmen einer systematischen Literaturanalyse, bei der sie 54 Studien berücksichtigten, 10 Dimensionen herausgearbeitet, die für Mobile Seamless Learning (MSL) als konstitutiv erachtet werden (2011: 8-9):

1. Lernaktivitäten, die sowohl formale als auch informelle Phasen bzw. Elemente aufweisen (formal - informell);

2. Lernaktivitäten, die personalisiertes und soziales Lernen umfassen (personalisiert sozial);

3. Lernaktivitäten, die sich über einzelne Zeitpunkte (z.B. Trainingstermine) hinaus erstrecken (zeitübergreifend);

4. Lernaktivitäten, die sich über verschiedene Orte hinweg erstrecken (ortsübergreifend); 
5. Lernaktivitäten, die Zugriff auf Informationen sowohl im Pull- als auch im Push-Modus beinhalten (push - pull);

6. Lernaktivitäten, die sich über physische und digitale Welten hinweg erstrecken (physisch - digital);

7. Lernaktivitäten, bei denen unterschiedliche Typen von Endgeräten (z. B. PC und Tablet) zum Einsatz kommen (unterschiedliche Endgeräte);

8. Lernaktivitäten, die mehrfache (und gegebenenfalls rasch aufeinanderfolgende) Wechsel zwischen verschiedenen Aufgaben (und damit u. U. Endgeräten) erfordern (Wechsel zwischen Aktivitäten);

9. Lernaktivitäten, die Wissenssynthese erfordern (z. B. die Integration von bisherigem und neuem Wissen; die Bewegung über verschiedene Stufen kognitiver Prozesse hinweg oder die Bearbeitung multidisziplinärer Fragestellungen [integrativ]);

10. Lernaktivitäten, die verschiedene didaktische Modalitäten bzw. Methodenformen umfassen (z. B. selbstgesteuertes und trainergeführtes Lernen oder instruktivistische und konstruktivistische Sequenzen) (vielfältig/variantenreich).

Diese 10 Dimensionen können in drei übergeordnete Bereiche eingegliedert werden, wie es Wong und Looi (2011: 24) in ihrer Meta-Studie zu Mobile Assisted Seamless Learning vorschlagen:

- Technologie (5 und 7)

- Pädagogik (8 und 10)

- Lernender (1-4, 6 und 9)

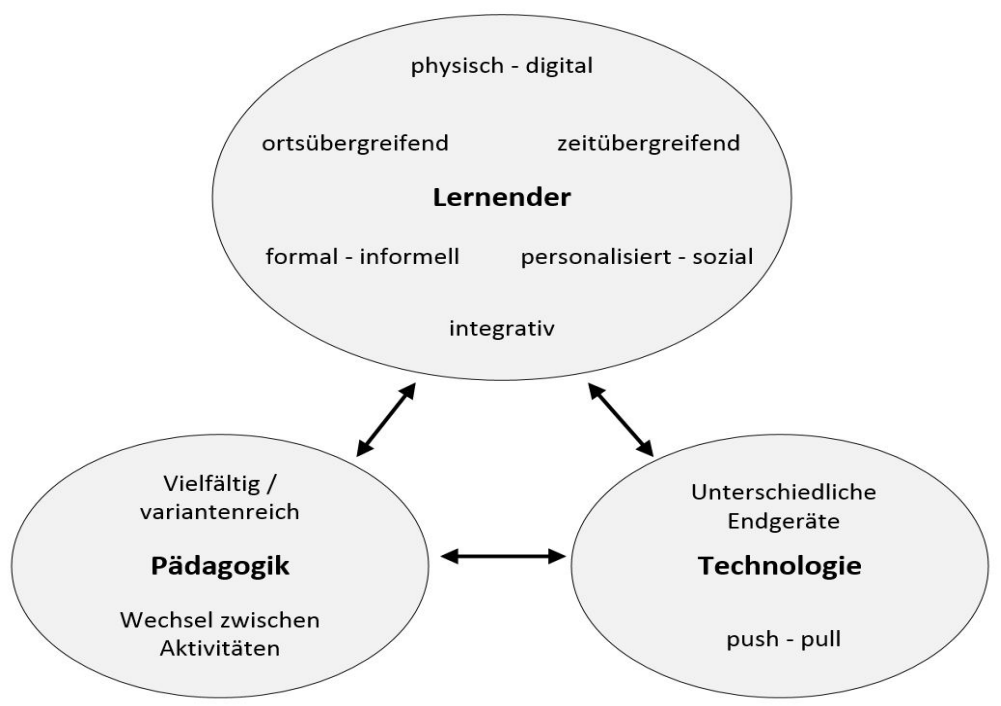

Abb. 710 Dimensionen von Seamless learning (Wong und Looi 2011; eigene Darstellung) 
Diese Zuordnung macht deutlich, dass der Lernende im Kontext von Mobile Assisted Seamless Learning immer stärker in den Mittelpunkt rückt. Im Zusammenspiel mit pädagogischen und technologischen Gestaltungselementen erhalten Lernende zunehmend Unterstützung bei Lernaktivitäten. Zudem können sie zwischen einer größeren Zahl an Lernoptionen wählen. Auf der anderen Seite steigen damit auch die Anforderungen an die Lernenden: einerseits im Hinblick auf die (persönliche) Ausstattung (Endgeräte, Zugang zu Netzwerken etc.), andererseits im Hinblick auf die Erwartung, die vielfältigen Möglichkeiten zur Kompetenzentwicklung tatsächlich auch zu nutzen.

\subsection{Empowerment-orientierte Organisationsentwicklung}

Das hier als Mobile Assisted Seamless Learning skizzierte integrierende Gesamtkonzept beinhaltet auch eine Abkehr von einem Denken in Kursen bzw. seminaristischen Angeboten und eine Hinwendung zu Empowerment-orientierter Organisationsentwicklung. Betriebliche Bildungsdienstleister müssen dabei die Interessen des Unternehmens einerseits und die der Mitarbeitenden andererseits in Abstimmung bringen. Betriebliches Bildungsmanagement ist zwar im Kern auf die systematische Gestaltung des Kompetenzentwicklungsprozesses der einzelnen Mitarbeitenden bzw. Lernenden ausgerichtet. Dabei geht es aber weniger um einzelne Lerneinheiten als vielmehr um die Gestaltung und Ermöglichung von größeren Entwicklungslinien. Diese größeren Entwicklungslinien können nur gelingen, wenn die Aktivitäten des Bildungspersonals einerseits und die Rahmenbedingungen für das Lernen in der Organisation („Lernkultur“) andererseits zusammenspielen. Die didaktische Lernorganisation (z.B. transferorientierte Funktionsweiterbildungen), die betriebliche Lernorganisation (z.B. Ermutigung zur Beteiligung an innerbetrieblichen Expertengemeinschaften und ErFA-Gruppen) sowie gegebenenfalls auch (informelle) Lernsituationen außerhalb des Betriebs (z. B. Austausch im Rahmen von Messen oder überbetrieblichen Fach- und Expertengruppen) müssen zusammenspielen. Dafür braucht es Lernkulturen, die auf Selbstorganisation und Empowerment ausgerichtet sind.

Auf diesem normativen Grundverständnis sollte nach unserer Ansicht ein betriebliches Bildungsmanagement aufbauen. Ohne Mobile Computing und Mobile Learning sind die damit verbundenen Herausforderungen nicht erfolgreich zu bearbeiten. „Alles wird mobil“ - so lautet ein Credo unserer Tage, und mobile Endgeräte (z. B. Smartphones und Tablets) werden zur wichtigsten Schnittstelle zum digitalen Ökosystem (MMB Institut 2016).

\section{$6 \quad$ Fazit und Ausblick}

Bei allem technischen Fortschritt im digitalen Zeitalter bleibt die Frage zu klären, wo der Mensch als Mitarbeiter oder Mitarbeiterin in dieser neuen Arbeitswelt seinen bzw. ihren Platz findet und wie dieser bestmöglich ausgefüllt werden kann. Eine auf die aktuellen 
Herausforderungen in Unternehmen und Organisationen ausgerichtete Kompetenzentwicklung, die Veränderungsbereitschaft, Agilität und Innovation in den Mittelpunkt stellt, erfordert mehr als nur formal organisierte Trainings. Es braucht ein breites Spektrum an Entwicklungsaktivitäten in dem durch die beiden Dimensionen „formal organisiertes Lernen - informelles Lernen“ und „individuelles Lernen - organisationales Lernen“ aufgespannten Möglichkeitsraum. Mobile Assisted Seamless Learning bietet sich hierzu als integrierendes Gesamtkonzept an:

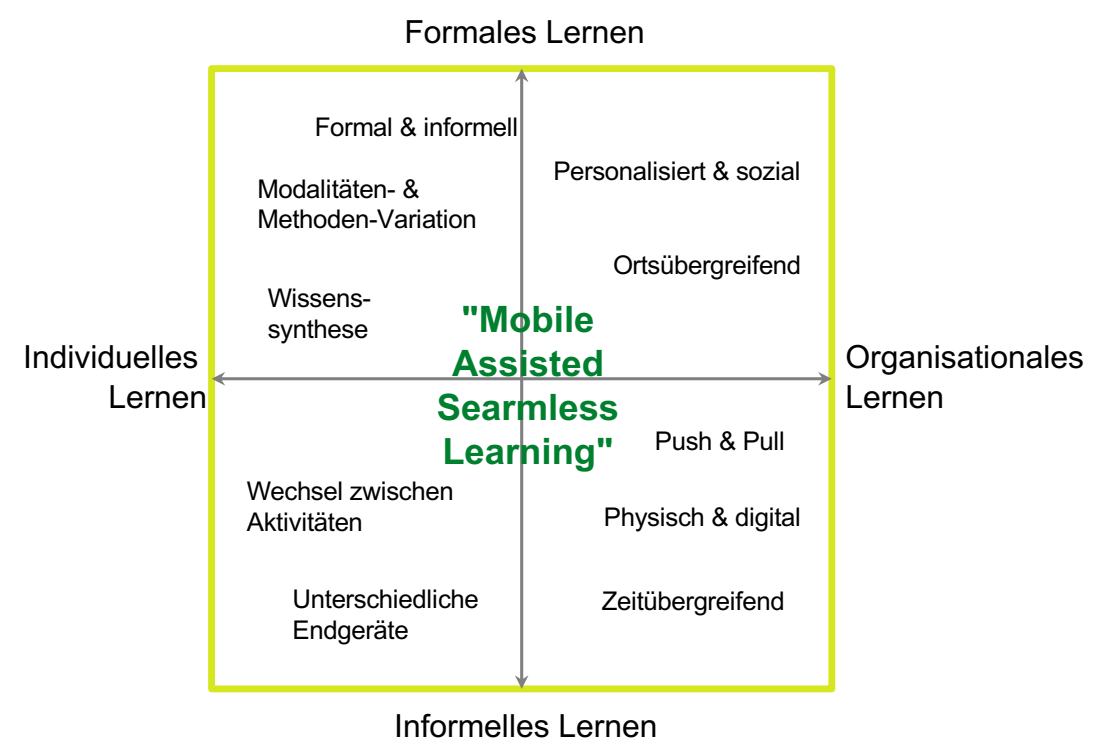

Abb. 8 Dimensionen von Mobile Assisted Seamless Learning im Ermöglichungsraum des betrieblichen Bildungsmanagements

Viele Organisationen wollen derzeit das Engagement ihrer Mitarbeitenden erhöhen, die unternehmensweite Kommunikation verbessern, eine „open innovation“-Kultur des Experimentierens fördern und sicherstellen, dass Veränderungen nachhaltig implementiert werden können - kurzum: eine neue Führungs- und Lernkultur für die künftige Arbeitswelt 4.0 prägen (Seufert und Meier 2016). Gemeinschaftliches, kollaboratives und wertschätzendes Lernen in Transformationsprozessen, die einen sinnstiftenden Zweck beinhalten, erfordern neue Ansätze der Kompetenzentwicklung in einem digitalen Ökosystem (Schuchmann und Seufert 2015). Ein derartiges Ökosystem schafft die notwendigen Rahmenbedingungen für mobiles Lernen in mehrerer Hinsicht (s. 10 Dimensionen oben). Das Einbeziehen der Mitarbeiter und Mitarbeiterinnen, insbesondere ermöglicht durch die mobile Konnektivität, stellt dabei ein oberstes Prinzip dar. Wie Empowerment-orientierte Personalentwicklung auf der Grundlage von „Mobile Assisted Seamless Learning“ konkret umgesetzt werden 
kann, das ist allerdings ein noch zu bearbeitendes Aufgabenfeld für Forschung und Entwicklung. Die Erfahrungen aus der Praxis (beispielsweise Zalando, Schweizer Post, SBB oder auch Swisscom) bieten ein empirisches Feld zur Analyse und Auswertung von „good practices" sowie zur weiteren Theoriebildung für das betriebliche Bildungsmanagement.

\section{Literatur}

Anderson, L. W., \& Krathwohl, D. R. (2001) (Eds). A Taxonomy for Learning, Teaching, and Assessing. A Revision of Bloom's Taxonomy of Educational Objectives. New York/NY.

Aufenanger, S. (2001). Multimedia und Medienkompetenz - Forderungen an das Bildungssystem. In S. Aufenanger, R. Schulz-Zander \& D. Spanhel (Hrsg.), Jahrbuch Medienpädagogik. Wiesbaden, 109-122.

Behrmann, D. (2006). Reflexives Bildungsmanagement. Pädagogische Perspektiven und managementtheoretische Implikationen einer strategischen und entwicklungsorientierten Gestaltung von Transformationsprozessen in Schule und Weiterbildung. Frankfurt a.M.

Beutner, M., \& Pechuel, R. (2012). Acceptance, Chances, and Problems of Mobile Learning in Vocational Education in Enterprises. In M. Specht, M. Sharples \& Jari Multisilta (Eds.), Proceedings of the 11th International Conference on Mobile and Contextual Learning, mLearn 2012, Helsinki, Finland, 16th-18th Octobre, 2012, 190-196.

Decker, J., Wesseloh, H., \& Schumann, M. (2015). Anforderungen an mobile Micro Learning Anwendungen mit Gamification-Elementen in Unternehmen. HMD Praxis der Wirtschaftsinformatik 52 (6), 851-865.

eLearning Journal (2015). Benchmarking Studie 2015. http://www.elearning-journal.de/index. php?id=1969. Zugegriffen: 17. Februar 2017.

Euler, D., \& Hahn, A. (2014). Wirtschaftsdidaktik. Bern.

Friedrich, H. F., \& Mandl, H. (1997). Analyse und Förderung des selbstgesteuerten Lernens. In F. E. Weinert \& H. Mandl (Hrsg.), Psychologie der Erwachsenenbildung. Göttingen, 237-293.

Harriehausen-Mühlbauer, B., \& Sonne, U. (2013). Lufthansa Privacy Quiz - Mobile Gaming für Smartphones. In C. de Witt \& A. Sieber, Mobile Learning. Wiesbaden, 241-262.

Hug, T. (2010). Mobiles Lernen. In K.-U. Hugger \& M. Walber (Hrsg.), Digitale Lernwelten. Wiesbaden, 192-211.

Jan, S., Ullah, F., Ali, H., \& Khan, F. (2016). Enhanced and Effective Learning through Mobile Learning - An Insight into Students Perception of Mobile Learning at University Level. International Journal of Scientific Research in Science, Engineering and Technology 2 (2), 674-681.

Kremer, H. H., \& Pferdt, F. G. (2008). Social Media Design - Grundlegung, Realisierungsformen und Gestaltungsattribute einer designorientierten Didaktik.bwp@15, 1-24.

Lave, J., \& Wenger, E. (1991). Situated Learning. Legitimate Peripheral Participation. Cambridge.

Meier, C. (2005). Gestaltungsfelder und Perspektiven für mobiles Lernen in der Hochschule. In D. Euler \& S. Seufert (Hrsg.), E-Learning in Hochschulen und Bildungszentren. München, 405-422.

Michel, L. P. (2014). Der Mittelstand baut beim e-Learning auf Fertiglösungen. Repräsentative Studie zu Status quo und Perspektiven von e-Learning in deutschen Unternehmen. Institut für Medienund Kompetenzforschung (MMB). Essen.

MMB Institut (2016). Schlussbericht zur Trendstudie. Digitale Bildung auf dem Weg ins Jahr 2025. Im Rahmen des Jubiläums 25 Jahre LEARNTEC - digitale Lernkultur im Wandel. Essen. 
https://www.learntec.de/data/studie-zur-25.-learntec/schlussbericht_zur_studie_digitale_bildung_auf_dem_weg_ins_jahr_2025.pdf. Zugegriffen: 15. Mai 2017.

Müller, C., Stahl, M., Lübcke, M., \& Alder, M. (2016). Flexibilisierung von Studiengängen: Lernen im Zwischenraum von formellen und informellen Kontexten. ZFHE: Zeitschrift für Hochschulentwicklung 11 (4), 93-107.

Robertson, R. (2017). Entwicklung und Design einer Mobile-Learning-Applikation für die Schulung afrikanischer Krankenhausmitarbeiter. In M. A. Pfannstiel, P. Da-Cruz \& H. Mehlich (Hrsg.), Digitale Transformation von Dienstleistungen im Gesundheitswesen. Wiesbaden, 257-269.

Schuchmann, D., \& Seufert, S. (2015). Corporate Learning in Times of Digital Transformation: A Conceptual Framework and Service Portfolio for the Learning Function in Banking Organisations. iJAC: International Journal of Corporate Learning 8 (1), 31-39.

Seufert, S. (2013). Bildungsmanagement. Einführung für Studium und Praxis. Stuttgart.

Seufert, S., Jenert, T., \& Kuhn-Senn, A. (2012). Didaktische Potenziale des Mobile Learning für die Berufsausbildung. Erfahrungen aus einem Pilotprojekt am Center for Young Professionals in Banking (CYP) in der Schweiz. BWP: Berufsbildung in Wissenschaft und Praxis 3 (41), 10-13.

Seufert, S., \& Meier, C. (2016). From eLearning to Digital Transformation: A Framework and Implications for L\&D. iJAC: International Journal of Corporate Learning 9 (2), 27-33.

Seufert, S., Meier, C., Schneider, C., Schuchmann, D., \& Krapf, J. (2017). Geschäftsmodelle für inner- und überbetriebliche Bildungsanbieter in einer zunehmend digitalisierten Welt. In J. Erpenbeck \& W. Sauter (Hrsg.), Handbuch Kompetenzentwicklung im Netz. Stuttgart, 429-447.

Shareground (2015). Arbeit 4.0: Megatrends digitaler Arbeit der Zukunft - 25 Thesen. Ergebnisse eines Projekts von Shareground und der Universität St. Gallen. https://www.telekom.com/resource/ blob/314922/.../dl-150902-studie-st--gallen-data.pdf. Zugegriffen: 15. Februar 2017.

Specht, M., \& Ebner, M. (2011). Mobiles und ubiquitäres Lernen - Technologien und didaktische Aspekte. In M. Ebner \& S. Schön (Hrsg.), Lehrbuch für Lernen und Lehren mit Technologie. Bad Reichenhall. http://13t.tugraz.at/index.php/LehrbuchEbner10/article/view/74. Zugegriffen: 05. April 2012.

Wentworth, D. (2014). Mobile Learning Research [CLO Webinar]. http://de.slideshare.net/humancapitalmedia/the-evolution-ofmobilelearning. Zugegriffen: 15. Februar 2017

de Witt, C. (2012). Mobile Learning - prozessbezogenes Informieren und Lernen in wechselnden Arbeitsumgebungen. Hagen: FernUniversität in Hagen. http://mlearning.fernuni-hagen.de/ wp-content/uploads/2013/06/1-BMBF-Fkz01PF07039A.pdf. Zugegriffen: 09. Dezember 2017

Wong, L.-H. (2012). A learner-centric view of mobile seamless learning. British Journal of Educational Technology 43 (1), E19-E23.

Wong, L.-H., \& Looi, C.-K. (2011). What seams do we remove in mobile assisted seamless learning? A critical review of the literature. Computers \& Education 57 (4), 2364-2381.

Wong, L.-H., Milrad, M., \& Specht, M. (2015). Seamless learning in the age of mobile connectivity. Singapore. 